\title{
CORES OR CUSPS IN ELLIPTICAL GALAXIES: LUMINOSITY
}

\section{OR ENVIRONMENT?}

\author{
ROELOF S. DE JONG, ROGER L. DAVIES, ROBERT F. MINCHIN, \\ JOHN R. LUCEY AND JAMES STEEL \\ Univ. of Durham, Dept. of Physics, South Road, Durham, UK
}

\section{The emerging view of galaxy cores}

Two classes of elliptical galaxies are now recognised (Kormendy \& Bender 1996). Luminous ellipticals rotate slowly (Davies et al. 1983and tend to have boxy isophotes. Ellipticals fainter than $L^{*}$ exhibit an increasing tendency to be rotationally supported and to possess a stellar disk component. This dichotomy led Bender, Burstein \& Faber (1992) to suggest that the physical variable that controls the ultimate nature of a forming galaxy is the degree of gaseous dissipation that occurs in the final merger it experiences. Low luminosity systems experience more dissipative mergers which generate high rotation, disky end products. As bigger galaxies are formed, the mergers become increasingly stellar, producing the classical slow rotating ellipticals. They termed this the gas/stellar continuum. This global dichotomy is also reflected in the bimodality of core morphologies of the heterogeneous sample of local ellipticals observed with HST. The low luminosity disky galaxies have 'hard' cores with a steep slope in the luminosity profile at small radii, whereas the luminous galaxies have 'soft' cores with flat profiles at small radii (e.g. Faber et al. 1997).

\section{Coma Cluster cores}

In the gas/stellar continuum paradigm, one would expect that in the outer parts of a cluster it is more likely that the last merger was gaseous than in its central regions. Therefore, at fixed luminosity, there should be more galaxies with 'hard' cores in the halo of the cluster than in the cluster centre. To test this hypothesis, we used WFPC-2 on the HST to survey a complete magnitude limited sample of ellipticals in the core of Coma, 




Figure 1. The slope of the luminosity profile at $0.015 \mathrm{R}_{e}$ as function of absolute $\mathrm{V}$-magnitude of the galaxy. The Coma core sample is indicated by circles, the Coma halo sample by squares and the Faber et al. (1997) sample by crosses. Upper limits are indicated by arrows for some dusty galaxies and for galaxies with $R_{e}<3.5^{\prime \prime}$.

together with a representative sample drawn from the cluster halo, to determine the environmental rôle in shaping cores. Fourteen out of the 46 galaxies show clear dust lanes and rings (evenly distributed among cluster centre and halo galaxies), and we have not considered these in our analysis.

We used a non-parametric method similar to that used by Gebhardt et al. (1996) to investigate the inner slope of the luminosity profile. Figure 1 shows this slope, $-\partial \log (I) / \partial \log (r)$ at $0.015 \mathrm{R}_{e}$, as function of magnitude for all samples. The distribution of the inner slope parameter of the Coma centre and the Coma halo sample are very similar. The same holds true for any other comparison of core parameters. Apparently, environment has no influence on determining core morphology.

The Coma sample also shows no appreciable bi-modality in Fig. 1, contrary to the Faber et al. (1997) sample, which has a clear deficiency of galaxies with $-\partial \log (I) / \partial \log (r)=0.45-0.7$. Our slope parameter is largely resolution independent, so the fact that the Faber et al. sample is on average much nearer (but observed with the pre-refurbished HST) should not effect our results. It seems unlikely that the Coma and Nuker samples are drawn from populations with the same intrinsic core properties.

\section{References}

Bender R., Burstein D., Faber S. 1992, ApJ 399, 462

Davies R.L., Efstathiou G., Fall S.M., Illingworth G., Schechter P.L. 1983, ApJ 266, 41

Faber S., et al. 1997, astro-ph/961055

Gebhardt K., et al. 1996, AJ 112, 105

Kormendy J., Bender R. 1996, ApJL 469, L119 